\title{
AGREEMENT BETWEEN PANORAMIC RADIOGRAPHY AND CBCT IN INTERPRETATION AND DIAGNOSIS OF JAW LESIONS
}

\author{
Raghdaa A. Mostafa*, Mostafa S. Ashmawy ${ }^{* *}$ and Walaa M. Hamed ${ }^{* * *}$
}

\begin{abstract}
Objective: this study aims to assess the rate of agreement between panoramic radiography (PR) and $\mathrm{CBCT}$ in evaluating the radiographic features of intraosseous lesions and detecting whether these differences had a significant impact on the accuracy of differential diagnoses.
\end{abstract}

Methods: Twenty four (24) intraosseous biopsy-proven lesions were reviewed using PR and CBCT images by two oral and maxillofacial radiologists. Both observers answered eighteen questions for each case describing the lesions` radiographic criteria and listed two possible differential diagnoses for each case.

Results: McNemar's test was used for comparing the two modalities and for calculating the interobserver agreement. The intra-observer agreement showed that questions 18 (cortical destruction) and 10 (expansion in the anatomical landmarks) had the lowest percentage of agreement between both observers. Furthermore, there was no statistically significant difference between the two modalities in terms of the agreement between differential diagnosis and final diagnosis $(\mathrm{P}>0.05)$.

Conclusions: Both PR and CBCT provide similar agreement in describing the lesion location, epicenter, internal structure. Moreover, similar agreement was also observed regarding the effect of the lesion on the teeth including resorption, impaction and displacement, root resorption, lamina dura, and periodontal membrane space of the teeth. The main disagreement between the two modalities was found in the expansion of the normal surrounding anatomic boundaries, and cortical destruction.

KEYWORDS: Cone-beam computed tomography; panoramic radiography; diagnosis.

\footnotetext{
* Lecturer of Oral and Maxillofacial Radiology, Faculty of Dentistry, Ain Shams University, Cairo, Egypt.

** Assistant Professor of Oral and Maxillofacial Radiology, Faculty of Dentistry, Ain Shams University, Cairo, Egypt.

*** Assistant Professor of Oral and Maxillofacial Radiology, Faculty of Dentistry, Ain Shams University, Cairo, Egypt
} 


\section{INTRODUCTION}

Interpretation of odontogenic and non-odontogenic lesions in the maxillofacial regions can be demanding because their clinical features may be nonspecific or may be detected incidentally. Although the clinical examination is essential, solid knowledge of their imaging appearance as morphology and extensions is essential in narrowing the range of differential diagnosis or permitting definitive diagnosis in some cases. ${ }^{1,2,3}$

Preoperative radiological evaluation defines the location of lesions and their extent within the jaws, which provide clues about the lesion characteristics and behaviors. This allows the surgeons to determine the appropriate management plan for the patient, follow up, and monitor for recurrence of disease or healing. Furthermore, it helps in avoiding surgical complications, postsurgical functional impairment, and reduces surgical stress. ${ }^{3}$

Due to the wide availability, ease of use, and low radiation dose, conventional radiographs such as panoramic radiographs (PR) form the backbone in the diagnosis of space-occupying lesions in the jaws. They can provide information about the internal structures of the lesions and even may provide a final diagnosis in some cases ${ }^{2}$

A previous study has demonstrated that PR has similar accuracy to computed tomography (CT) when measuring well-defined lesions located in the posterior region of the mandible. The PR technique is also considered a suitable method for evaluating odontogenic cystic lesions in the mandible. However, in the maxilla, it is difficult to assess lesions that are close to the maxillary sinus using a twodimensional (2D) image. ${ }^{4}$

Cone-beam computed tomography (CBCT) is an important imaging tool for oral and maxillofacial diagnosis of osseous lesions with the advantages of providing three dimensional (3D) information similar to CT. CBCT also allows image manipulation and reconstruction in non-orthogonal planes. ${ }^{5}$
Although the advantages of CBCT are indisputable, the oral radiologist needs to identify these benefits and understand the additional information which is not available using PR. Also, it is essential to notice the radiographic signs which might or might not appear when using CBCT compared to PR. Finally, it is crucial to know if the use of CBCT would increase the accuracy of differential diagnoses or affect the confidence of oral radiologists in their diagnoses. ${ }^{3}$

The aim of this study is to evaluate if the radiographic features of intraosseous lesions differ between PR and CBCT and whether these differences had a significant impact on the accuracy of differential diagnoses.

\section{MATERIALS AND METHODS}

\section{Sample collection}

Our retrospective comparative study was exempted from the Review of the Ethics Committee as all included PR (panoramic radiograph) and CBCT were retrieved from the records of the Oral and Maxillofacial Radiology Department, Faculty of Dentistry, Ain Shams University. Thirty-eight (38) patients with an age range from five to fifty years old were selected from the database of the Oral and Maxillofacial Radiology Department. The selected patients had undergone both PR (panoramic radiograph) and CBCT examinations (between July 2019 and December 2019) for diagnosis and/or treatment planning of pathological space-occupying lesions in the maxillofacial region. The patients' medical history, dental history, and clinical criteria were retrieved from their archived records as well as the final diagnosis confirmed by histopathologic examination.

All cases were selected under with the following inclusion criteria: 1) A maximum of a one-month interval between PR and CBCT examinations 2); Clear visibility of the lesion in both PR and CBCT; 3) The biopsy confirmed a pathological lesion. On 
the other hand, cases were excluded if the dental or medical records were incomplete, if the lesion was extending to soft tissue, if any surgical procedure was performed between PR and CBCT or if the biopsy result was inconclusive. Finally, twenty-four patients $(\mathrm{N}=24)$ were included in this study with the age range from twenty-six to fifty years (Age 26-50 y).

\section{Radiographic Examination}

Panoramic images were selected from the database of the department's panoramic machine PaX-i (Vatech, Geonggi, South Korea) which uses the following parameters for adult panoramic acquisition: $70 \mathrm{kVp}, 10 \mathrm{~mA}$, and $13 \mathrm{sec}$ scanning time. The CBCT images were selected from the database of the department's CBCT machine i-CAT next generation (Imaging Sciences International, Hatfield, PA). All the patients' personal data were removed from the images and the images were coded with numbers by the primary investigator to make the observers blinded to the patients' data.

\section{Image Analysis}

Two oral radiologists (with 16 and 17 years of experience) reviewed the panoramic images independently in a darkened room on a 21 " DELL Flatron monitor (DELL, Precision T79110 XL, United States) with a screen resolution of $1920 \times$ 1200 pixels and 64-bit color depth. The observers were blinded to all the patients' data. Both observers had no time limit for viewing the images and they were allowed to adjust brightness, contrast, and to use the zoom tool. Both observers answered eighteen questions for each case as shown in Figure 1. The questions were designed to describe the lesions' radiographic criteria and listed two possible differential diagnoses for each case. After two weeks both observers reviewed the CBCT images independently using the i-CAT Vision software (Imaging Sciences International, Hatfield, PA) in a darkened room on the same monitor but with a different order of the cases to prevent any possible bias. They were allowed to navigate through different MPR (multiplanar reformatting) slices,

\begin{tabular}{|c|c|c|c|c|}
\hline & Question & \multicolumn{3}{|c|}{ Answer } \\
\hline 1 & Describe the Location of the lesion & A. Localized in the mandible & B. Localized in the maxilla & C. In maxilla and mandible \\
\hline 2 & The lesion center is in which region & $\begin{array}{l}\text { A. Incisors / canine region } \\
\text { B. Premolar/molar region }\end{array}$ & $\begin{array}{l}\text { C. Ramus region } \\
\text { D. Maxillary sinus }\end{array}$ & E. Multifocal \\
\hline 3 & What is the relation of the teeth to the Lesion & $\begin{array}{l}\text { A. Pericoronal } \\
\text { B. Periapical }\end{array}$ & $\begin{array}{l}\text { C. Inter radicular } \\
\text { D. Missing tooth Associated }\end{array}$ & $\begin{array}{l}\text { E. No direct relation to } \\
\text { teeth }\end{array}$ \\
\hline 4 & Describe the lesion's shape & A. Round/ovoid & B. Scalloped & C. Irregular \\
\hline 5 & The periphery of the lesion is & A. Well defined & B. Ill defined & C. Not certain \\
\hline 6 & If the periphery of the lesion is well defined, is it & $\begin{array}{l}\text { A. Defined and corticated } \\
\text { B. Defined but not corticated - } \\
\text { Sclerosis }\end{array}$ & $\begin{array}{l}\text { C. Defined but not corticated } \\
\text {-No bony reaction } \\
\text { D. Defined but not corticated } \\
\text { - Capsulated }\end{array}$ & E. Not applicable \\
\hline 7 & If the periphery of the lesion is ill defined, is it & $\begin{array}{l}\text { A. Ill-defined outlines showing } \\
\text { signs of invasion }\end{array}$ & $\begin{array}{l}\text { B. Ill-defined outlines } \\
\text { showing blending margin }\end{array}$ & C. Not applicable \\
\hline 8 & The internal structure of the lesion is & $\begin{array}{l}\text { A. Radiolucent } \\
\text { B. Mixed with bonelike density }\end{array}$ & $\begin{array}{l}\text { C. Mixed with tooth-like } \\
\text { density }\end{array}$ & D. Radiopaque \\
\hline 9 & $\begin{array}{l}\text { If the internal structure of the lesion is radiolucent, is the } \\
\text { lesion multilocular? }\end{array}$ & A. Yes & B. No & C. Not applicable \\
\hline 10 & $\begin{array}{l}\text { Does the lesion appear to expand the normal surrounding } \\
\text { anatomic boundaries? }\end{array}$ & A. Yes & B. No & C. Not applicable \\
\hline 11 & $\begin{array}{l}\text { Does the lesion appear to be affecting the inferior alveolar } \\
\text { canal? }\end{array}$ & $\begin{array}{l}\text { A. Yes } \\
\text { B. No }\end{array}$ & C. Not certain & $\begin{array}{l}\text { D. Not applicable (in } \\
\text { another location) }\end{array}$ \\
\hline 12 & $\begin{array}{l}\text { Does the lesion appear to be affecting the maxillary sinus } \\
\text { or/ and nasal cavity? }\end{array}$ & $\begin{array}{l}\text { A. Yes } \\
\text { B. No }\end{array}$ & C. Not certain & $\begin{array}{l}\text { D. Not applicable (in } \\
\text { another location) }\end{array}$ \\
\hline 13 & Is there subperiosteal new bone formation? & A. Yes & B. No & C. Not certain \\
\hline 14 & Does the lesion appear to be causing tooth displacement? & $\begin{array}{l}\text { A. Yes } \\
\text { B. No }\end{array}$ & C. Not certain & $\begin{array}{l}\text { D. Not applicable (in } \\
\text { another location) }\end{array}$ \\
\hline 15 & Does the lesion appear to be causing root resorption? & $\begin{array}{l}\text { A. Yes } \\
\text { B. No }\end{array}$ & C. Not certain & $\begin{array}{l}\text { D. Not applicable (in } \\
\text { another location) }\end{array}$ \\
\hline 16 & Does the lesion appear to be causing tooth impaction? & A. Yes & B. No & \\
\hline 17 & $\begin{array}{l}\text { Does the lesion appear to be affecting the lamina dura or } \\
\text { the periodontal membrane space of the teeth? }\end{array}$ & A. Yes & B. No & C. Not certain \\
\hline 18 & Is there cortical destruction? & A. Yes & B. No & C. Not certain \\
\hline
\end{tabular}

Fig. (1) Questionnaire applied for analysis of intra-osseous lesions 
adjust brightness, contrast, and magnification. The observers answered the same questions in Figure 1 and listed two possible differential diagnoses for each case. Answer sheets were collected for statistical evaluation.

\section{Statistical Analysis}

Qualitative data were presented as frequencies and percentages. McNemar's test was used for comparing the two modalities and for calculating the inter-observer agreement. The significance level was set at $\mathrm{P} \leq 0.05$. Statistical analysis was performed with IBM SPSS Statistics for Windows, Version 23.0. Armonk, NY: IBM Corp.

\section{RESULTS}

Cases included in the present study were 11 odontogenic cysts, 8 benign odontogenic tumors, two malignant tumors, two fibro-cemento-osseous lesions, and one case was diagnosed as central giant cell granuloma. A summary of the cases is presented in Table 1.

TABLE (1) Summery of the cases included in the present study

\begin{tabular}{|c|c|c|}
\hline Category & Diagnosis & Number \\
\hline \multirow{3}{*}{ Cysts $(n=11)$} & Periapical Cyst & 7 \\
\hline & Residual Cyst & 2 \\
\hline & Dentigerous Cyst & 2 \\
\hline \multirow{5}{*}{$\begin{array}{l}\text { Benign Tumors } \\
\qquad(\mathrm{n}=8)\end{array}$} & Ameloblastoma & 2 \\
\hline & Odontogenic Keratocyst & 1 \\
\hline & Odontogenic Myxoma & 1 \\
\hline & Cemento-Ossifying Fibroma & 2 \\
\hline & Complex/ Compound Odontoma & 2 \\
\hline $\begin{array}{c}\text { Malignant } \\
\text { Tumors }(n=2)\end{array}$ & Squamous Cell Carcinoma & 2 \\
\hline \multirow{2}{*}{ Others $(n=3)$} & Central Gaint Cell Granuloma & 1 \\
\hline & Fibro- Osseous Lesion & 2 \\
\hline
\end{tabular}

Each observer evaluated the same case using both PR and CBCT images. Intra-observer agreement showed that, for observer (1) question 13 had the highest percentage of agreement (100\%) followed by an equal percentage $(95.83 \%)$ for questions 1,3 , and 16 while questions 18 and 10 had the lowest percentage of agreement $(54.17 \%$ and $33 \%$ respectively). For observe (2) questions 1, 8, and 16 had an equal high percentage of agreement (95.83\%) while questions 18 and 10 had the lowest percentage of agreement $(37.50 \%$ and $12.5 \%$ respectively).

The inter-observer agreement showed that, pertaining PR modality; the highest inter-observer agreement was found with questions number 1, 16 then 13 (95.83\%, $95.83 \%$, and $91.67 \%$, respectively) and the lowest agreement was found with questions number 4,6 then $15(54.17 \%, 54.17 \%$, and $45.83 \%$, respectively). On the other hand, in the $\mathrm{CBCT}$ modality, the highest inter-observer agreement was found with questions number 18, 1 then 16 (100\%, $95.83 \%$ and $95.83 \%$, respectively). The lowest agreement was found with questions number 14, 15 than $6(70.83 \%, 66.67 \%$ and $29.17 \%$, respectively).

With respect to the agreement between differential diagnosis and final diagnosis and based on Observer 1 findings; it was found that agreement between differential diagnosis and final diagnosis with PR and CBCT modalities were $70.83 \%$ and $79.17 \%$, respectively. There was no statistically significant difference between the two modalities $(\mathrm{P}$-value $=0.500$, Effect size $=0.799)$.

With regards to Observer 2 findings, it was observed that agreement between differential diagnosis and final diagnosis with PR and CBCT modalities were $75 \%$ for each modality. There was no statistically significant difference between the two modalities $(\mathrm{P}$-value $=1.000$, Effect size $=$ $1.000)$. 


\section{DISCUSSION}

A wide spectrum of pathologies occurs in the oral and maxillofacial area. Some of them may be restricted to the bony tissues, or to the soft tissue, others may include both tissues. Diagnostic imaging is considered to be an essential step that contributes to the diagnosis with the histopathology being the gold standard upon which crucial decisions are taken regarding patient management ${ }^{6}$

To the best of the authors' knowledge, few studies ${ }^{3,4,7}$ evaluated the difference in interpretation between CBCT and PR for intraosseous maxillofacial lesions and correlated it with histopathology. Therefore, this study attempted to investigate whether CBCT provides more information than panoramic images for interpretation of lesions that can increase the accuracy of differential diagnosis by oral and maxillofacial radiologists.

In the present study, a series of questions regarding the key features of the lesions were conveyed. The first three questions were related to the location of the lesion and the point at which the lesion originated (the epicenter of a lesion). The results of the present study showed equal agreement between both modalities. These results are expected since the location of the lesion tends to be obvious. Yet, this data is important as it might provide information about the nature of the tissue involved within the lesion. ${ }^{8,9}$

With regards to the shape of the lesion, the results showed $75 \%$, and $58.33 \%$ agreement rate between PR and CBCT for observers 1 and 2 respectively. These percentages are in agreement with a study by Lim et al., ${ }^{3}$. The authors found an overall substantial agreement $($ Kappa value $=0.65)$ between PR and CBCT for the same feature. The variations in the degree of agreement between both modalities are perhaps due to the presence of multiple superimpositions in the PR, especially in the mid-face and sinus regions. ${ }^{10}$
With regards to the periphery of the lesion, high agreement was observed between the two modalities in determining whether the lesion is well or ill-defined. In contrast, Lim et al. ${ }^{3}$ reported that lesions are better defined on CBCT compared with panoramic radiographs. The authors attributed their results due to the differences in image acquisition. In CBCT, the details can be seen due to the lack of the superimposing structures present in the panoramic radiograph. It should be mentioned that different questions regarding this feature were asked in Lim et al. ${ }^{3}$ and ours.

Concerning evaluating the internal structure of the lesion whether radiolucent, radiopaque, or mixed radiolucent-radiopaque, high agreement between the PR and CBCT images for both observers was found. Similar results were reported by Lim et $\mathrm{al}^{3}$. This was in contrast to the findings report by Chindasombatjaroen et al., ${ }^{11}$ on a case report of a calcifying cystic odontogenic tumor. The authors revealed that the detailed internal pattern of calcifications could not be seen on a PR image and were only visible on CBCT.

Several articles reported the importance of CBCT in identifying mixed density lesions because of the variable appearance of internal calcifications. ${ }^{12}$, 13 The difference between our results and the previous studies may be related to the high percentage of lesions with radiolucent internal structures in our study sample. Larger numbers of lesions with mixed internal structures are needed for better assessment of the value of CBCT compared to PR.

A moderate agreement was found for assessing the multilocularity of lesions between both modalities. Koçak-Berberoğlu et al., ${ }^{14}$ evaluated 4 cases of keratocystic odontogenic tumors using panoramic and CBCT images. The authors reported that only one lesion that appeared as unilocular in the panoramic view was later reported to be multilocular in CBCT images. Koong ${ }^{9}$ stated that the presence of bone septa within the lesions does not necessarily mean that this corresponds to a multilocular pattern. 
Evaluating the effect of a lesion on the surrounding structures helps in describing the behavior of the lesion ${ }^{9}$. In the current study, PR and CBCT images showed agreement regarding teeth displacement, tooth resorption, effect on lamina dura and periodontal ligament space. Lim et al., ${ }^{3}$ reported significant agreement between PR and CBCT concerning tooth displacement. The authors stated that these results are expected as tooth displacement tends to be obvious radiographically.

The only questions that showed the lowest agreement between the two modalities in the current study were about the expansion of the anatomical boundaries and cortical bone destruction. These two features are correlated, as when the lesion expands in size to the inferior border of the mandible, it may cause perforation or destruction. ${ }^{3}$ Cortical bone destruction may be masked on PR by an intact buccal or lingual cortical plate. In contrast, CBCT reveals cortical thinning and/or destruction. It also provides valuable information for surgical treatment planning about the presence and the direction in which expansion is occurring $3,11,13,15$

Concerning the diagnostic impact of the two imaging modality, the results of the present study showed no significant difference in providing the correct differential diagnosis for both PR and CBCT when compared to histopathological diagnosis which was in agreement with Lim et al., ${ }^{3}$. The authors found no correlation between the confidence of the observers in their differential diagnosis and either modality.

Limitations of this study include the final sample size which was only limited to 24 cases with $45 \%$ of the cases in the category of odontogenic cysts. This might lead to the non-significant difference between both panoramic and CBCT imaging modalities. Another limitation of this study is that the interpretation of the lesions only depended on radiographic images, providing clinical information may improve the accuracy of reaching a correct diagnosis.

\section{CONCLUSIONS}

Close agreement between PR and CBCT was observed for describing the lesion location, epicenter, and internal structure. Moreover, PR and CBCT modalities provide similar agreement concerning the effect of a lesion on the teeth including resorption, impaction and displacement, root resorption, lamina dura, and periodontal membrane space of the teeth. Low agreement between the two modalities was mainly found in the expansion of the normal surrounding anatomic boundaries, cortical destruction.

\section{Financial support and sponsorship}

Nil.

\section{Conflicts of interest}

There are no conflicts of interest.

\section{REFERENCES}

1. Razek AAKA (2011). Imaging appearance of bone tumors of the maxillofacial region. World J Radiol. 3(5): 125-134

2. Avril L, Lombardi T, Ailianou A, Burkhardt K, Varoquaux A, Scolozzi P, et al (2014). Radiolucent lesions of the mandible: a pattern-based approach to diagnosis. Insights Imaging. Feb; 5(1): 85-101.

3. Lim LZ, Padilla RJ, Reside GJ, Tyndall DA (2018). Comparing panoramic radiographs and cone beam computed tomography: Impact on radiographic features and differential diagnoses. Oral Surg Oral Med Oral Pathol Oral Radiol. ;S2212-4403(18)30888-5.

4. Alves DBM, Tuji FM, Alves FA, Rocha AC, Santos-Silva ARD, Vargas PA et al (2018) . Evaluation of mandibular odontogenic keratocyst and ameloblastoma by panoramic radiograph and computed tomography. Dentomaxillofac Radiol. 47(7):20170288

5. Shweel M, Amer MI, El-shamanhory AF (2013). A comparative study of cone-beam CT and multidetector CT in the preoperative assessment of odontogenic cysts and tumors. The Egyptian Journal of Radiology and Nuclear Medicine. 44, 23-32

6. Almazrooa SA, Binmadi NO, Khalifa HM, Jadu FM, Jan AM, Meisha DE (2019). The agreement rate between radiographic interpretation and histopathologic diagnosis of jaw lesions. Radiology Research and Practice.: 2019, 1-5. 
7. Weiss R 2nd, Read-Fuller A (2019). Cone Beam Computed Tomography in oral and maxillofacial surgery: An Evidence-Based Review. Dent J (Basel).7(2):52.

8. Neyaz Z, Gadodia A, Gamanagatti S, Mukhopadhyay S (2008). Radiographical approach to jaw lesions. Singapore Med J.49(2): 165-177.

9. Koong B (2012). The basic principles of radiological interpretation. Aust Dent J. 57(s1)(3)33-39

10. Perschbacher S (2012). Interpretation of panoramic radiographs. Aust Dent J. 57:(1 Suppl): 40-45

11. Chindasombatjaroen J, Poomsawat S, Boonsiriseth $\mathrm{K}$ (2014). Two unique cases of calcifying cystic odontogenic tumor in the maxillary posterior region. Oral Surg Oral Med Oral Pathol Oral Radiol. 118:497-504.

12. Marques YMFS, Botelho TDL, Xavier FCDA, Rangel AL, Rege ICC, Mantesso A (2010). Importance of cone beam computed tomography for diagnosis of calcifying cystic odontogenic tumor associated to odontoma. Report of a case. Med Oral Patol Oral Cir Bucal. 15:1-4

13. Luo J, You M, Zheng G, Xu L (2014). Cone beam computed tomography signs of desmoplastic ameloblastoma: review of 7 cases. Oral Surg Oral Med Oral Pathol Oral Radiol. 118:e126-e133.

14. Koçak-Berberoğlu H, Çakarer S, Brkić A, Gürkan-Koseoglu B, Altuğ-Aydil B, Keskin C (2012). Three-dimensional cone-beam computed tomography for diagnosis of keratocystic odontogenic tumours; evaluation of four cases. Med Oral Patol Oral Cir Bucal. 1; 17(6):e1000-5.

15. Treister NS, Friedland B, Woo SB (2010). Use of conebeam computerized tomography for evaluation of bisphosphonate-associated osteonecrosis of the jaws. Oral Surg Oral Med Oral Pathol Oral Radiol Endod. 109(5):753-764. 